\title{
A brief review on vessel extraction and tracking methods
}

\begin{abstract}
Extracting an accurate skeletal representation of coronary arteries is an important step for subsequent analysis of angiography images such as image registration and 3D reconstruction of the arterial tree. This step is usually performed by enhancing vessel-like objects in the image, in order to differentiate between blood vessels and background, followed by applying the thinning algorithm to obtain the final output. Another approach is direct extraction of centerline points using exploratory tracing algorithm preceded by a seed point detection schema to provide a set of reliable starting points for the tracing algorithm. A large number of methods fall in these two approaches and this paper aims to contrast them through a brief review of their innate characteristics, associated limitations and current challenges and issues.
\end{abstract}

Keyword: Coronary artery; Exploratory tracing; Seed point detection; Tracking; Vessel centerline extraction 